\title{
ARTICLE
}

\section{A peculiar dehydration and solid-solid phase transformation of the active pharmaceutical ingredient AZD9898 based on in situ single crystal-to-single crystal transformations}

Received 00th January 20xx, Accepted 00th January 20xx DOI: $10.1039 / x 0 x x 00000 x$

\author{
Anna Pettersen, ${ }^{* a}$ Okky Dwichandra Putra, ${ }^{b}$ Mark E. Light, ${ }^{c}$ and Yukiko Namatame ${ }^{d}$
}

\begin{abstract}
AZD9898 has previously been used as a candidate for a potential active pharmaceutical ingredient. AZD9898 Form A hydrate was discovered during development and this form undergoes dehydration upon heating to give anhydrous Form $B$. Further heating results in a solid-solid phase transformation to a new anhydrous phase, Form $C$. The crystal structures of Forms $A$, $B$, and $C$ were obtained by heating the single crystal of Form A in situ on the diffractometer allowing establishment of dehydration and solid-solid phase transformation mechanisms. The relevant thermal profiles and vapour sorption behaviour of these forms are also reported in this study.
\end{abstract}

\section{Introduction}

Understanding the solid form landscape of active pharmaceutical ingredients (APIs) has become a major endeavour for pharmaceutical companies and academia given that each solid form will have different physicochemical properties such as solubility, stability and bioavailability. ${ }^{1,2}$ Indeed, exploration of new solid forms, such as polymorphs, solvates, hydrates, amorphous, co-crystals, salts, or any combination thereof is an important aspect in pharmaceutical research and development. The aim of the solid form exploration is to achieve an understanding of which specific form will be best suited for further development and commercialisation. ${ }^{3-7}$ It is also important to assess the solid form and the solid form landscape of APIs to establish reliable control strategies to avoid phase transformation or dissociation during manufacturing processes or upon storage of the drug in its final dosage form. ${ }^{8,9}$

Water molecules are commonly incorporated into a crystal structure along with API molecule to form hydrates. ${ }^{10}$ Notably, around $30 \%$ of drugs listed in the European Pharmacopeia can exist as hydrates. ${ }^{11}$ It is well known that environmental changes, i.e., variation in temperature or humidity can induce structural changes leading to dehydration of pharmaceutical hydrates during drug manufacture. The anhydrous form of a compound

a. Early Product Development and Manufacturing, Pharmaceutical Sciences, BioPharmaceuticals R\&D, AstraZeneca, Gothenburg, Pepparedsleden 1, Mölndal SE-431 83, Sweden.Email: anna.pettersen@astrazeneca.com

b. New Modality and Parenteral Development, Pharmaceutical Technology and Development, AstraZeneca Gothenburg, Pepparedsleden 1, Mölndal SE-431 83, Sweden.

c. UK National Crystallography Service, School of Chemistry, Faculty of Engineering and Physical Sciences, University of Southampton, SO17 1BJ Southhamptonn, UK. d. Rigaku Corporation, Sendagaya 4-14-4, Shibuya-ku, Tokyo 151-0051, Japan.

+ Electronic Supplementary Information (ESI) available: crystallographic details, DVS, and XRD-DSC measurements. CCDC 1982526-1982528. For ESI and crystallographic data in CIF or other electronic format see DOI: 10.1039/x0xx00000x may well have different physicochemical properties compared to its parent hydrate. ${ }^{12-15}$ Consequently, understanding the dehydration of pharmaceutical hydrates is an issue of considerable importance in API development.

Generally it is a prerequisite to understand the structural properties prior to evaluating the mechanistic aspect of dehydration of pharmaceutical hydrates. ${ }^{16}$ Dehydration mechanisms are often explained from the crystal structure differences between hydrate and corresponding anhydrous phases. Subtle changes in the crystal structure usually correspond to simple dehydration and significant reorganization of crystal structures correlate with more complex dehydration processes. ${ }^{17,18}$ For this reason, it is important to determine the crystal structures both for hydrated and anhydrous forms. Also, the structural differences between hydrated and anhydrous crystal structures can be used to rationalize the differences in physicochemical properties. ${ }^{19}$

Among many available techniques used to determine crystal structures, X-ray diffraction, which includes powder diffraction and single crystal structure determination, is arguably the most commonly used technique. In the most cases, single crystals of the initially hydrated phase begin to lose integrity and transform to a polycrystalline phase; structure determination using powder methods are therefore usually employed to establish the dehydration mechanism of hydrated APIs. ${ }^{20-24}$. Systems where the hydrated phase retains its single-crystallinity upon dehydration are more experimentally challenging. As a result, there are only a few examples reported where the dehydration mechanism is established using single crystal X-ray structure determination. ${ }^{25,26}$ Herein we report just such a 


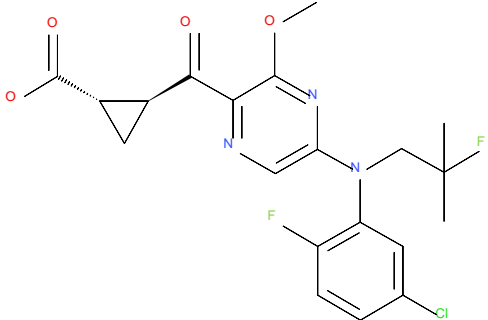

Fig. 1 Chemical structure of AZD9898.

rare system which undergoes single-crystal-to-single-crystal dehydration and phase transformation.

AZD9898 (Fig. 1) was previously used as a potential drug candidate and was identified as a picomolar LTC4S enzyme inhibitor with high lipophilic potency. ${ }^{27}$ Hydrate crystals of AZD9898 was identified during early development. In this study, we aim to reveal the structural changes during dehydration by using AZD9898 as a model compound to clarify the underlying mechanism of dehydration from the hydrate form to an anhydrous form. Structure elucidation of hydrate and anhydrous forms are unequivocally indispensable to provide understanding of dehydration process on a molecular level. Therefore, we employed single crystal X-ray diffraction which is arguably the most advanced technique for structural characterization.

\section{Experimental}

\section{Materials}

AZD9898 was synthesized according to previously published method. This material was assigned to have purity more than $99.9 \%$ and was used as received. ${ }^{27}$ The other chemicals and solvents employed in this study were of analytical grade and used without any purification.

\section{Methods}

Powder X-ray diffraction (PXRD). PXRD measurements were performed using XPert X-Ray diffractometer (PANalytical, Netherlands). The measurements were carried out in reflection mode. The powder pattern was collected from $2 \theta=3^{\circ}$ to $50^{\circ}$ at $25^{\circ} \mathrm{C}$ with a step and measurement time of $0.01^{\circ}$ and 1 hour, respectively (Cu Ka source, 45 kV, $40 \mathrm{~mA}$ ).

Thermal gravimetric analysis (TGA) and differential scanning calorimetry (DSC) analysis. TGA and DSC measurements were performed using a TG Discovery 550 (TA instruments, Germany) and DSC Discovery 2500 (TA instruments, Germany), respectively. Approximately $10 \mathrm{mg}$ for TGA and 2-3 mg of the sample for DSC were weighed into an aluminium pan. The samples were then heated from room temperature to $350^{\circ} \mathrm{C}$ and from $-50^{\circ} \mathrm{C}$ to $200^{\circ} \mathrm{C}$ for TGA and DSC, respectively, at a heating rate of $3{ }^{\circ} \mathrm{C} \mathrm{min}{ }^{-1}$ under a nitrogen purge of $100 \mathrm{~mL} \mathrm{~min}{ }^{-1}$. An empty aluminium pan was used as a reference. Open and closed pans were used for TGA and DSC measurements, respectively. Modulated mode was used for DSC measurement with modulation temperature amplitude and modulation period set at $1{ }^{\circ} \mathrm{C}$ and $60 \mathrm{~s}$, respectively.

Dynamic vapour sorption (DVS). Vapour sorption isotherms were measured by dynamic vapour sorption (DVS) at $25{ }^{\circ} \mathrm{C}$ using a Dynamic Vapour Sorption Resolution instrument (Surface
Measurement System, UK). Samples were pre-sieved prior to mounting in a balance and the relative humidity $(\mathrm{RH})$ was increased from 0 to $80 \%$ and decreased from 80 to $0 \%$ during the first and second cycle, respectively, in $5 \%$ steps. The waiting time for $0.001 \%$ weight change was set to $30 \mathrm{~min}$ and the further step was increased or decreased automatically.

Single crystal $X$-ray diffraction and structure refinements. Single crystals of AZD9898 were grown by vapour diffusion technique using isopropylacetate/ $n$-heptane as solvent/antisolvent. A suitable single crystal was mounted on an AFC12 FRE-VHF diffractometer (Rigaku, Japan) equipped with an Oxford Cryosystems Cobra (Oxford Cryosystem, UK). Data were measured using Mo $K_{\alpha}$ radiation generated from a rotating anode source $(45.0 \mathrm{kV}, 55.0 \mathrm{~mA})$. Measurement strategy, data reduction, and data correction were performed using the CrysAlisPro ${ }^{28}$ software (Rigaku, Japan). Using Olex $2,{ }^{29}$ the structure was solved with the ShelXS ${ }^{30}$ structure solution program using the direct methods solution method. The model was refined on $F_{0}{ }^{2}$ with ShelXL 2014. ${ }^{31}$ All non-hydrogen atoms were refined anisotropically. Hydrogen atom positions were calculated geometrically and refined using the riding model, and the $\mathrm{O}-\mathrm{H}$ were located from the difference map and refined using distance restraints of $0.84 \AA$ and thermal parameters set to be 1.5 times those of the parent atom.

Simultaneous X-ray diffraction- differential scanning calorimetry (XRD-DSC). Combined XRD-DSC measurements were performed using a SmartLab X-ray diffractometer (Rigaku, Japan). The sample was placed in a flat aluminium pan. The powder pattern was collected from $2 \theta=5^{\circ}$ to $40^{\circ}$ with a step and scan speed of $0.01^{\circ}$ and $35^{\circ} \mathrm{min}^{-1}$, respectively. The heating speed for DSC was set to be $1^{\circ} \mathrm{C} \mathrm{min}-1$. A rotating anode $\mathrm{Cu} K \alpha$ source was employed with the $\mathrm{X}$ ray power set to $45 \mathrm{kV}, 100 \mathrm{~mA}$.

\section{Results and Discussion}

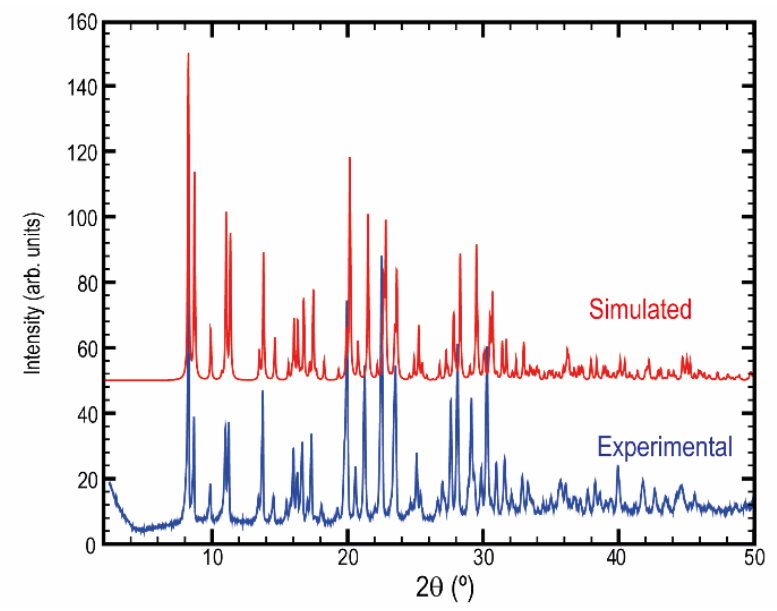

Fig. 1 Experimental and simulated powder patterns of AZD9898 Form A hydrate. 
(a)

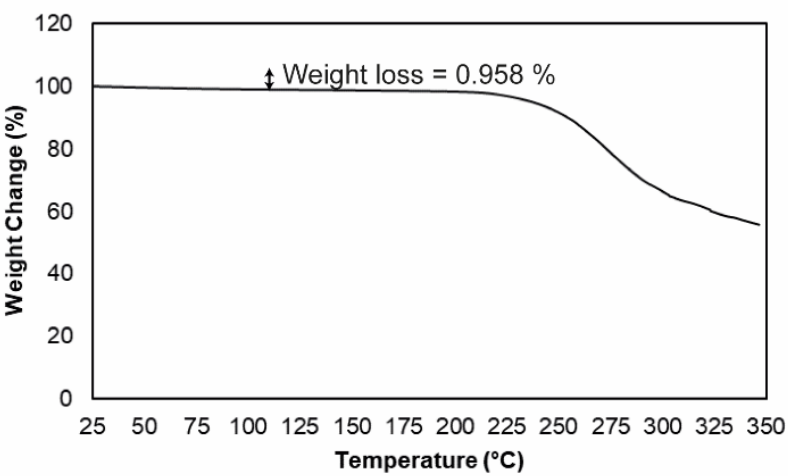

(b)

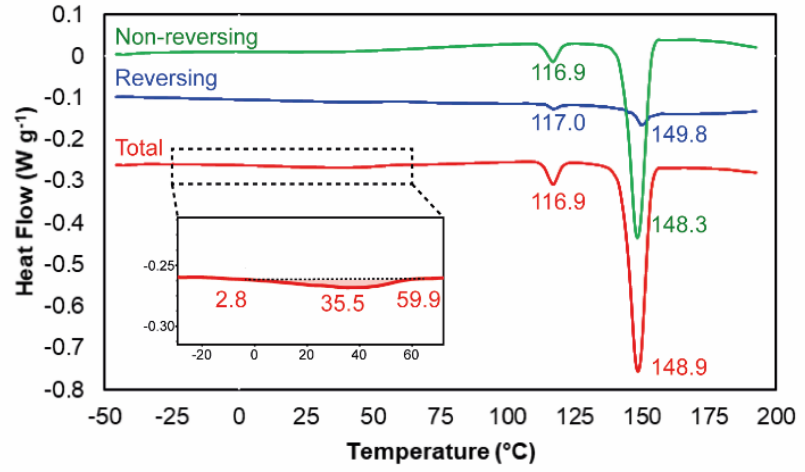

Fig. 3 (a) TGA and (b) DSC scans of AZD9898 Form A hydrate. The red, blue, and green lines in DSC thermogram show total, reversing, and non-reversing heat flows, respectively. Inset shows a zoomed in view of the dehydration in (b).

\section{AZD9898 Form A hydrate}

A polymorph screen was performed on AZD9898 using various solvents, temperatures, and crystallization techniques during early phase development. The single crystal structure of the methanol solvate of AZD9898 (CSD Entry: SIXVIN) has previously been reported in the litterature. ${ }^{27}$ In the polymorph screen, a distinctive hydrate phase, Form A hydrate, was isolated from a slurry experiment at room temperature in isopropyl acetate solvent. However, a vapour diffusion technique was used to grow single crystals of AZD9898 Form A using isopropyl acetate as the solvent and $n$-heptane as the antisolvent. The powder pattern of the polycrystalline material isolated from the slurry experiment matches the simulated powder pattern from the single crystal analysis indicating that a pure phase is obtained (Fig. 2).

Thermal analysis, (TGA and DSC), were performed on freshly prepared material obtained by grinding single crystal samples. TGA and DSC analysis are expected to give a general understanding of the response of Form $A$ to heating. From the TGA, a slight weight change ( $0.958 \%)$, which corresponds to $\sim 0.25$ water molecule per AZD9898 molecule, is observed up to $100{ }^{\circ} \mathrm{C}$ (Fig. 3a). We attribute this weight loss to a dehydration of AZD9898 Form A hydrate. There is no weight change observed between 100 and $200{ }^{\circ} \mathrm{C}$. Further heating results in significant weight loss corresponding to the thermal decomposition.

DSC in modulated mode was chosen to characterize AZD9898 because of its ability to resolve complex transition behaviour, i.e., overlapped thermal events. ${ }^{32}$ In addition, DSC measurements were carried out from sub-zero temperature to minimize potential dehydration prior to data collection, i.e., during preconditioning and equilibration. A small broad endothermic peak is observed in the total heat flow DSC scan (red line in Fig. $3 \mathrm{~b}$ ) between 2.8 and $59.0^{\circ} \mathrm{C}$ with a maxima at $35.5^{\circ} \mathrm{C}$. This endothermic peak is assigned as the dehydration of AZD9898 Form A. It is interesting that dehydration starts below room temperature in AZD9898 Form A hydrate which implies the instability of the hydrate phase. The dehydration enthalpy is found to be $21.87 \times 10^{-3} \mathrm{~J} \mathrm{~mol}^{-1}$. Dehydration is completed at $59.9^{\circ} \mathrm{C}$ resulting in the anhydrous phase, namely Form $\mathrm{B}$.

Upon further heating, the DSC exhibits another endothermic transformation with a maximum observed around $117^{\circ} \mathrm{C}$. This endothermic peak is assigned to a solid-solid phase transformation. Therefore, the phase between the first and second endothermic temperature range is of Form $B$ anhydrous and after completion of the second endothermic peak, the new anhydrous phase exists until the last endothermic peak which corresponds to melting at $148.9^{\circ} \mathrm{C}$. This new anhydrous phase is referred to as AZD9898 Form C anhydrous. The phase transition of Form $B$ anhydrous to Form $C$ anhydrous upon heating is reversible, given that Form $C$ converts back to Form $B$ upon cooling.

Separation of the total heat flow in the DSC scan into reversing and non-reversing heat flow confirms that there are no other thermal events observed during ramping. Therefore, there are only three phases observed, Form A, Form B, and Form $C$ that are obtained during temperature ramping starting from AZD9898 Form A hydrate.

We observed that a crystalline powder of AZD9898 Form A hydrate changed to Form $B$ anhydrous during storage at room temperature $\left(22^{\circ} \mathrm{C}, 30 \%\right.$ relative humidity $\left.(\mathrm{RH})\right)$. Samples that have been stored under these conditions show no substantial weight loss prior to $100{ }^{\circ} \mathrm{C}$ as seen from TGA data and no endothermal event from the DSC data 
(a)

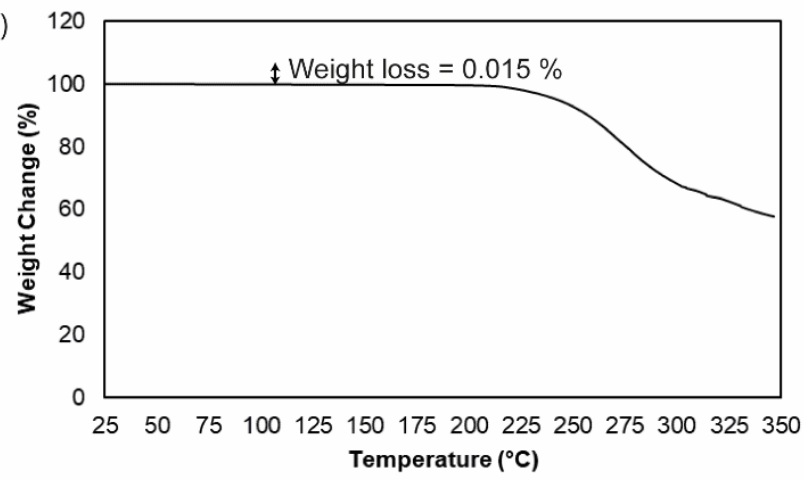

(b)

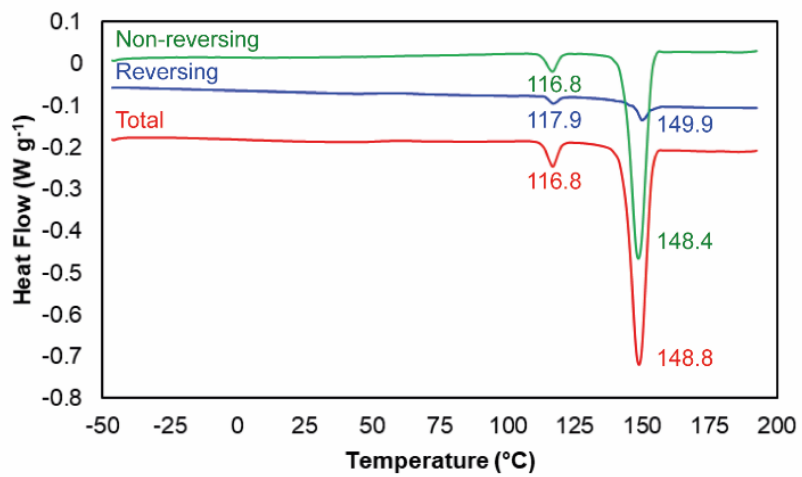

Fig. 4 (a) TGA and (b) DSC scans of AZD9898 Form A hydrate showing dehydration to Form B anhydrous. The red, blue, and green lines in the DSC thermogram indicate total, reversing, and non-reversing heat flows, respectively.

(Fig. 4). Notably, the phase transition profile from Form B anhydrous to Form $C$ anhydrous and the melting behaviour of Form C anhydrous remain similar. The instability of AZD9898 Form A hydrate is not surprising since the dehydration starts below room temperature (at $2.8^{\circ} \mathrm{C}$ ). Also, the PXRD measurements of freshly-ground single crystals of AZD9898 Form A hydrate do not differ significantly from the pattern of AZD9898 Form B anhydrous as can be seen in Fig. 5, which indicates that Form B anhydrous and Form A hydrate are isostructural. In other words, Form B anhydrous is the dehydrated form of Form $A$, and the easy dehydration of Form A hydrate to Form $B$ anhydrous can be explained by the isostructurality.

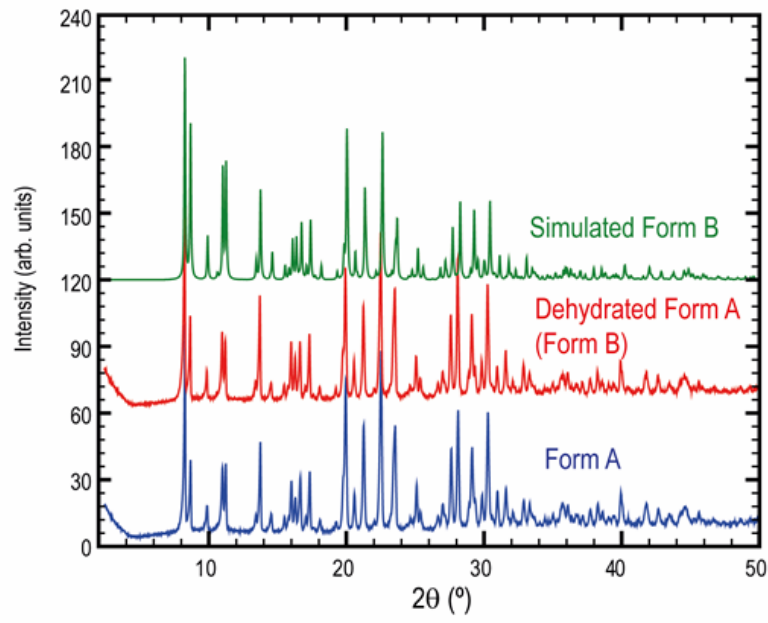

Fig. 5 PXRD pattern comparison of AZD9898 Form A hydrate dehydrated Form A (Form B) and simulated Form B.
Water activity, or the equilibrium of water vapour uptake as a function of vapour partial pressure is another important aspect for manufacture and development of pharmaceuticals. To investigate the water activity of AZD9898, we performed vapour sorption isotherms using DVS methods. ${ }^{33}$ As can be seen from the DVS chart of AZD9898 Form A in Fig. 6, there is a reversible conversion between AZD9898 Form A hydrate and Form $B$ anhydrous. Form $A$ hydrate is stable at $\mathrm{RH}>65 \%$ during sorption, and $>60 \%$ during desorption. On the other hand, Form $\mathrm{B}$ anhydrous is stable at $0 \% \mathrm{RH}$. Therefore, Form A and B could coexist between 5 to $60 \% \mathrm{RH}$ which cover most ambient conditions. It should be noted that the DVS chart of Form B does not differ significantly from Form A (Fig S1). The absence of a steep change in the DVS chart indicates that the water

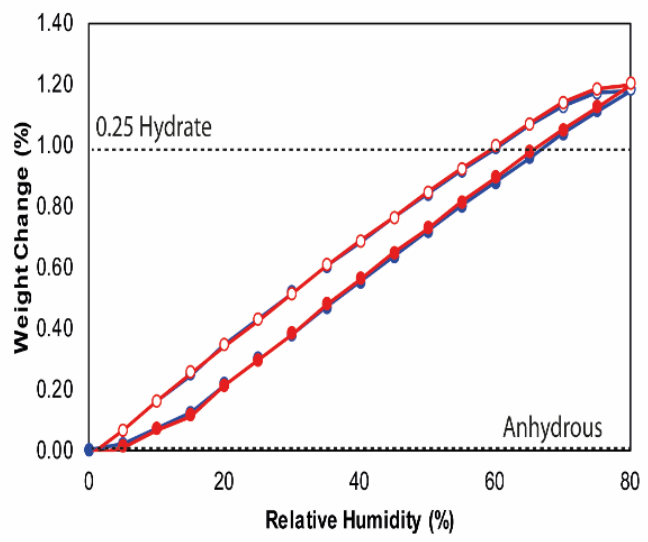

Fig. 6 DVS chart of AZD9898 Form A hydrate. Red and blue lines indicate the first and second cycle, respectively. Closed and open symbols represent sorption and desorption, respectively. Top and bottom dashed blacklines show theoretical mass where Form $A$ and B exist. 
Table 1 Crystallographic data of AZD9898 Form A, B, and C. $¥$

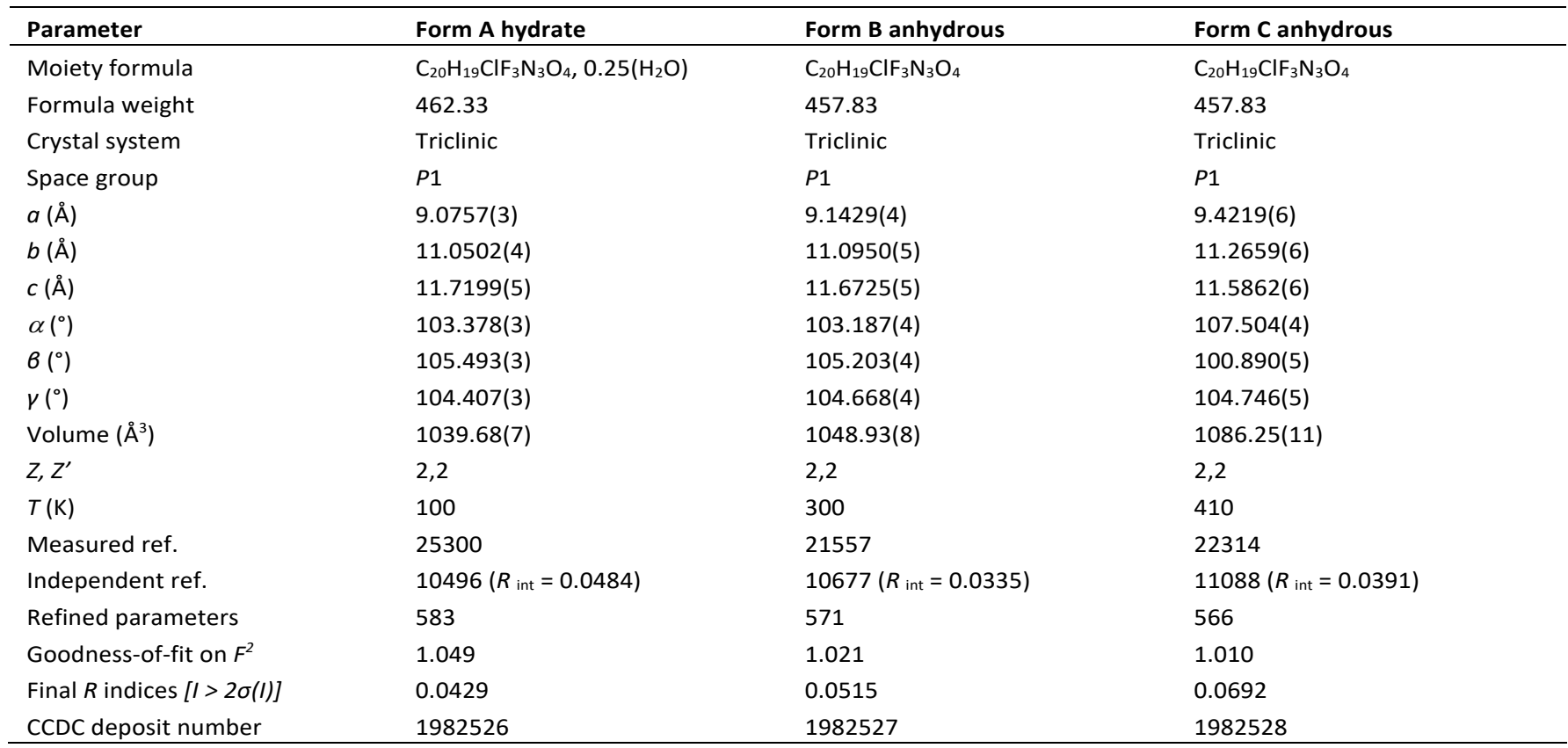

molecule in AZD9898 Form A can be easily absorbed/desorbed from the crystal structure which is frequently observed for nonstochiometric hydrates. This again suggests the easy dehydration of Form $A$ hydrate to Form $B$ anhydrous, as well as facile rehydration from Form $B$ anhydrous to Form $A$ hydrate.

\section{Crystal structure of AZD9898 Form A hydrate}

The crystallographic details of the three forms in this study, are listed in Table 1. Form A crystallises in the triclinic chiral space group $P 1$. There are two independent molecules of AZD9898, and one water molecule in the asymmetric unit. During crystal structure refinement, a significant residual density peak was observed in the difference Fourier map and this was assigned as the oxygen of a water molecule. Initially the site occupancy factor was freely refined and then fixed at the refined value of 0.5. Therefore, the stochiometric ratio between the AZD9898 molecule and water becomes 0.25 as there are two independent AZD9898 molecules, this is in good agreement with TGA and DVS data.

The nature of water molecules in hydrate crystals can be analysed using hydrate analyser within Mercury (CFC 4.1.1). ${ }^{34}$ As can be seen in Fig. 7, the water molecules are located in discrete pockets, while the DVS data shows that these crystals behave like a non-stoichiometric hydrate. Generally, nonstoichiometric hydrates display lattices where the water molecules are located in continuous pockets/channels instead of discrete pockets. The reason of the non-stochiometric behaviour of AZD9898 Form A hydrate could be seen from the thermodynamic aspect instead of solely from the crystal structure. As mentioned above, the dehydration enthalpy for AZD9898 Form A hydrate is $21.87 \times 10^{-3} \mathrm{~J} \mathrm{~mol}^{-1}$ from DSC measurements. This value is much smaller than the corresponding enthalpy value of the vaporisation of water $\left(\Delta_{\text {vap }} H^{\circ} \mathrm{H}_{2} \mathrm{O}=41.585 \mathrm{~kJ} \mathrm{~mol}^{-1}\right){ }^{35}$ Therefore, it is not surprising that dehydration is more favourable from a thermodynamic point of view and similar cases are reported for pyrogallol, barbituric acid, and phloroglucinol. ${ }^{36-38}$

In the structure of AZD9898 Form A hydrate, voids are observed in the crystal packing (yellow translucent space in Fig. 7). The unit cell contains voids of $24.4 \AA^{3}$, which is equivalent to $2.3 \%$ of the unit cell volume (using the Voids analyser in Mercury with 1.2 and $0.7 \AA$ probe radius and 0.7 approximate grid spacing, respectively). From a further development perspective, the presence of such voids should become a flag for a potential stability problem. The nature of this void can be considered as hydrophobic

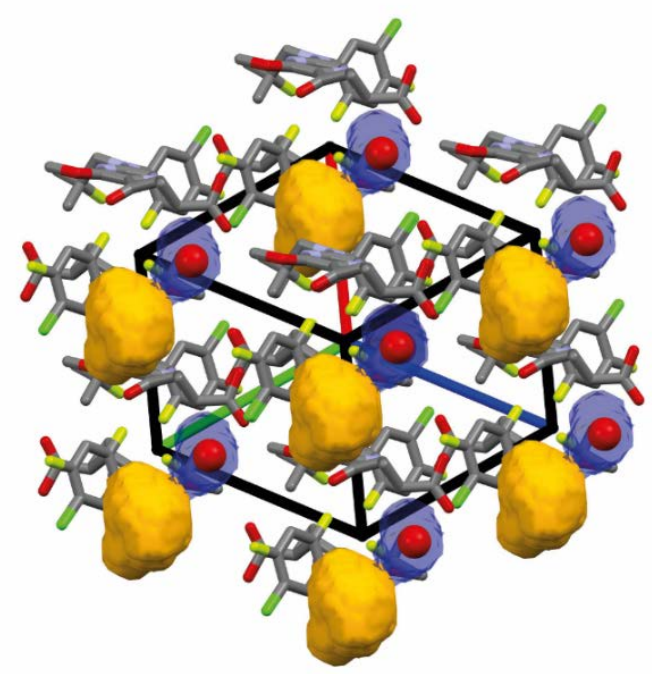

Fig. 7 Crystal packing of AZD9898 Form A hydrate. Hydrogen atoms are omitted for clarity. Water molecules are drawn as spheres of fixed radius. Blue and yellow translucent spaces indicate space occupied by water molecules and voids, respectively. 

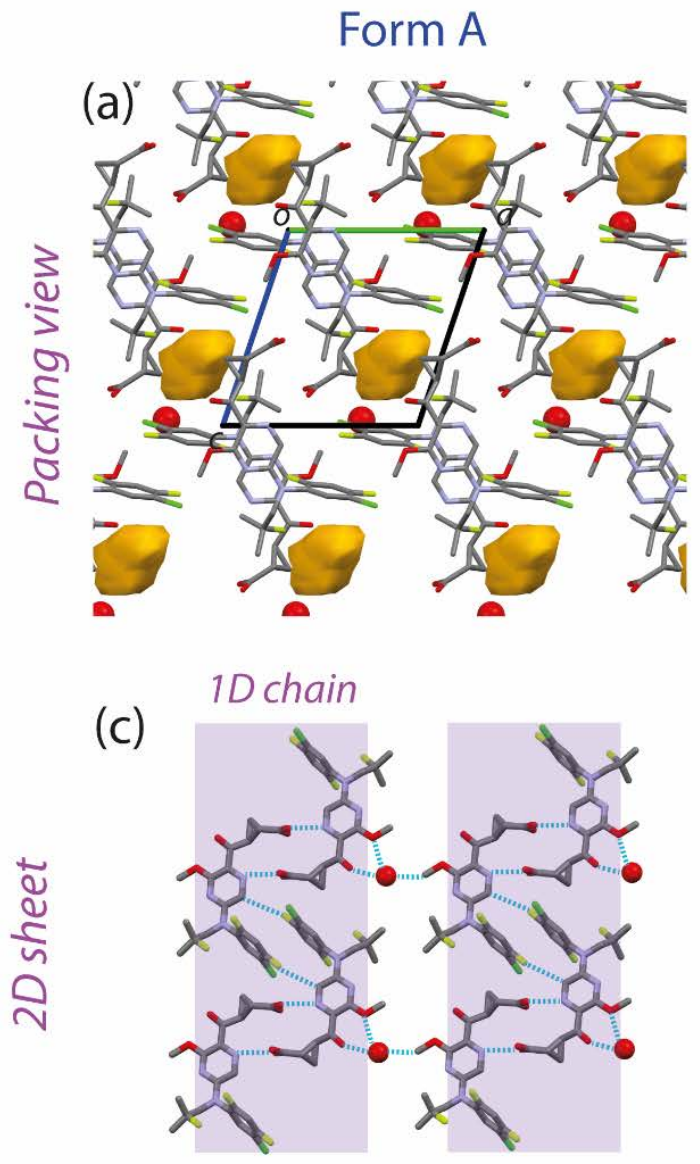

(e)

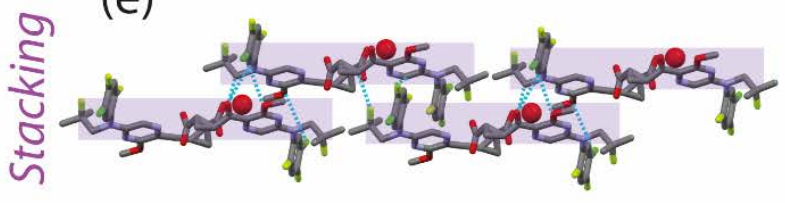

Form B

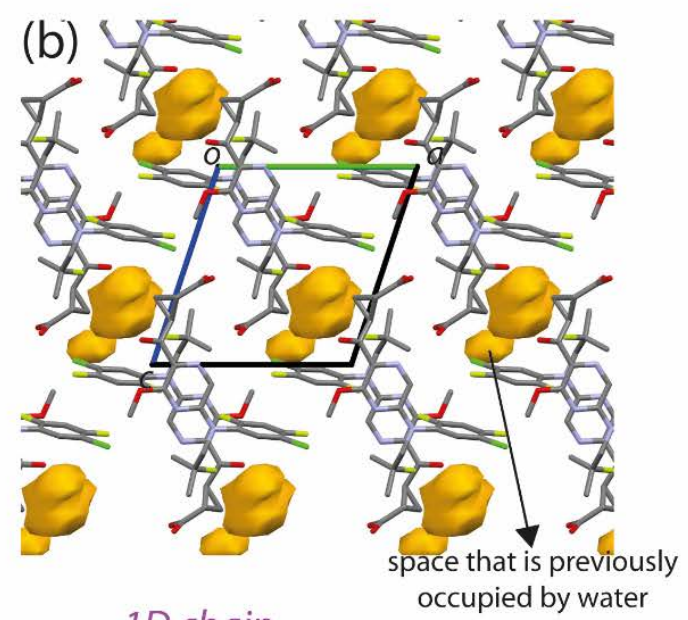

(d)

(f)

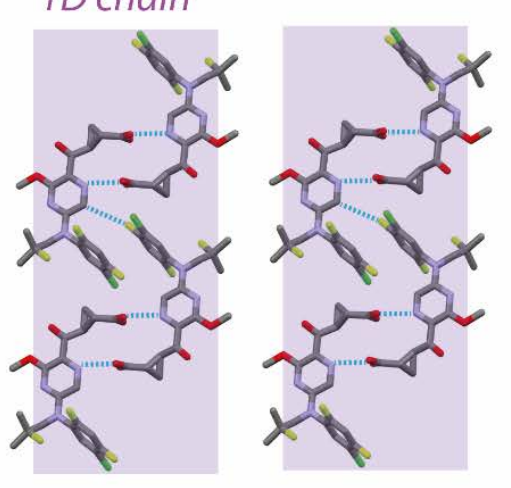

Fig. 8 Crystal structures of AZD9898 Form A hydrate (a, c, and e) and Form B anhydrous (b, d, and f). Hydrogen atoms are omitted for clarity. Water molecules in Form A are drawn as spheres of fixed radius. Blue dashed lines indicate hydrogen bonds. Yellow translucent spheres in (a) and (b) indicate voids.

as this void does not accommodate water molecules or any other solvents used during crystallisation.

\section{Crystal structure comparison of AZD9898 Form A and B and the dehydration mechanism}

To understand the mechanism of dehydration from AZD9898 Form A hydrate to Form B anhydrous, the crystal structures of these forms were analysed. Inspired by the fast dehydration of Form $\mathrm{A}$ as a bulk powder, we attempted to dehydrate a single crystal of Form $\mathrm{A}$ in situ on the single crystal X-ray diffractometer. The same single crystal of Form A was heated to $300 \mathrm{~K}$. Interestingly, the crystallinity of the single crystal was retained which enabled data collection at $300 \mathrm{~K}$ on the same crystal. During crystal structure refinement, no significant residual density was observed in the difference of Fourier map and it was not possible to refine a partial water molecule in this location. Therefore, it is convincing that single crystal of
AZD9898 Form A hydrate has dehydrated and transformed to Form $\mathrm{B}$ anhydrous at $300 \mathrm{~K}$.

AZD9898 Form B anhydrous still crystallises in the triclinic space group $P 1$ with only slight changes in the lattice parameters. The asymmetric unit in AZD9898 Form B also consists of two independent molecules. The expansion of the volume to $1048.93(8) \AA^{3}$ observed can also be caused by thermal expansion due to temperature change from 100 to 300 $\mathrm{K}$.

The crystal packing of AZD9898 Form A hydrate and Form B anhydrous are similar as seen in Fig. $8 a$ and $b$, and the crystals are isostructural. In the unit cell, there are two independent voids with the size of $25.34 \AA^{3}$ or about $2.4 \%$ of the total unit cell volume. The position of the smaller voids in Form $B$ and the water molecules in Form $A$ are almost the identical, meaning that these smaller voids are created when water molecules leave the crystal. As the smaller voids were occupied with water 
molecules, we deduce that this void is more hydrophilic than the larger voids which are more hydrophobic.

The hydrogen bond interactions described in this paper were determined by Platon. ${ }^{39}$ In AZD9898 Form A hydrate, the one-dimensional (1D) chain structure is connected to anadjacent $1 \mathrm{D}$ chain structure through the water molecule to create the so-called two-dimensional (2D) sheet structure (Fig. $8 \mathrm{C})$. Thess 2D sheets are stacked on top of each other to create the overall 3D packing of the crystal structure. Interestingly, the 1D chain structures in AZD9898 Form B anhydrous are not connected by hydrogen bonds but still retain a similar $2 \mathrm{D}$ sheet structure. We predict that weaker interactions such as van der Waals interactions play an important role in retaining the $2 \mathrm{D}$ sheet structure inthe absence of water linker.

No energetic stabilisation by hydrogen bonds is possible in Form B when water molecule leaves the structure. Therefore, only small structural changes are observed when the water molecules reversibly enter and leave the crystal explaining the non-stochiometric behaviour between form $A$ hydrate and Form B anhydrous.

\section{Solid-solid phase transformation to AZD9898 Form C}

The crystal structure of Form $C$ was analysed by further heating of the same single crystal of Form B to $410 \mathrm{~K}$ mounted on the single crystal X-ray diffractometer. Elaborating with DSC data discussed above, $410 \mathrm{~K}$ could be considered as a temperature point where the solid-solid phase transformation is expected to be complete. Interestingly, single crystallinity was still retained during this transition and there is no evidenceof amorphization, which is remarkable and unusual enabling data collection at 410 $\mathrm{K}$.

AZD9898 Form C also crystallises in triclinic space group $P 1$ and contains two independent molecules in asymmetric unit.

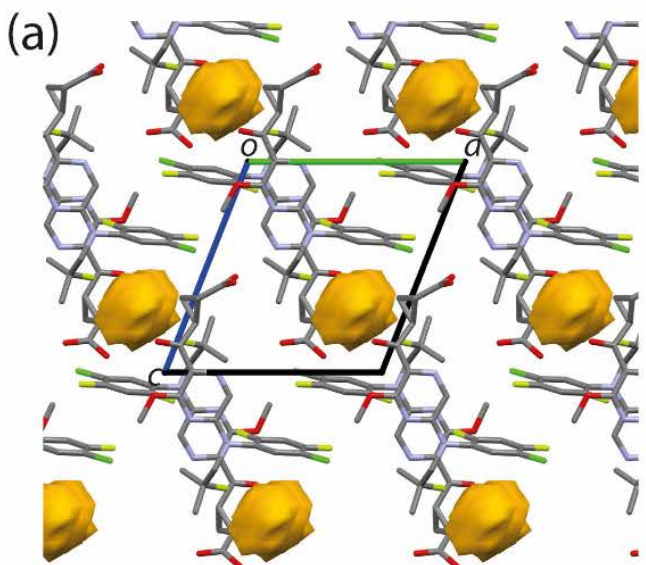

(b)

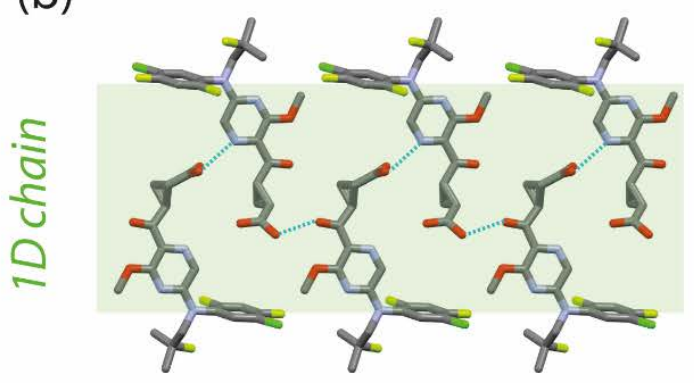

(c)

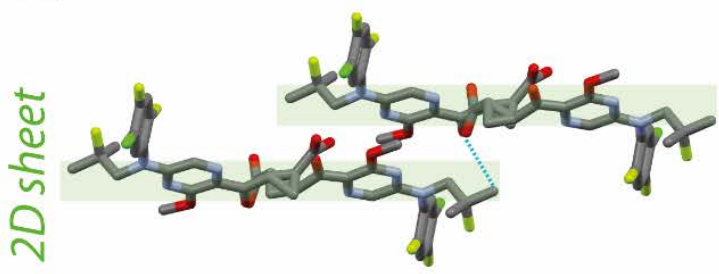

(d)

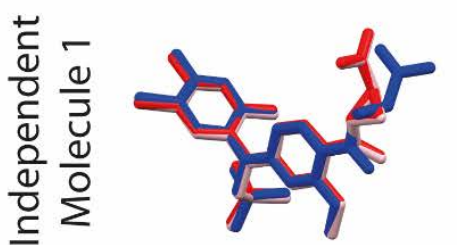

Form A

Form B

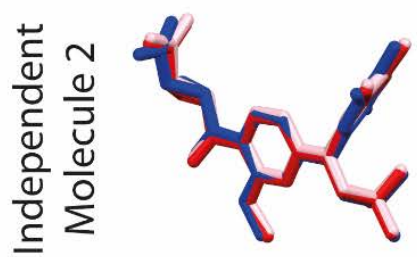

Form C

(e)

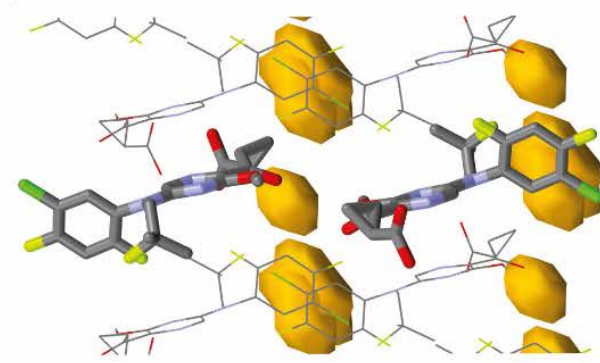

Conformation change + Hydrogen bond formation

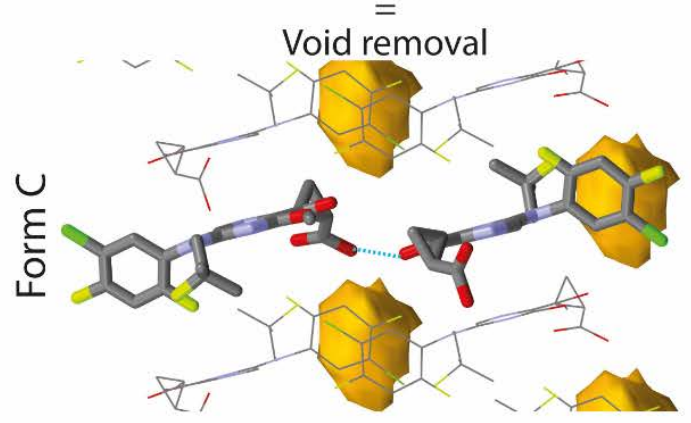

Fig. 9 (a) Packing view, (b) 1D chain and (b) 2D sheet structures of AZD9898 Form C anhydrous. Hydrogen atoms are omitted for clarity. Blue dashed lines indicate hydrogen bond. (d) Overlaid independent molecules in AZD9898 Form A, B and C showing significance conformational difference in Form C compared to Form A and B. (e) The proposed mechanism of smaller void removal in Form B when the crystal transformed to the Form C. Yellow translucent spheres indicate void. 
There are significant differences in the lattice parameters, especially the value of $\alpha$ and $B$, and therefore, AZD9898 Form C anhydrous is not isostructural with Form $A$ and $B$. The overall crystal packing of AZD9898 Form C anhydrous is shown in Fig. 9a. Form $\mathrm{C}$ has a different hydrogen bond network compared to Form A and Form B as can be seen in Fig. 9b and c, and only one independent void is observed in the lattice. The location of this void is similar to that of the larger hydrophobic void observed in Form A and Form B. The void in Form C occupies 2.5\% of volume of the lattice or $27.65 \AA^{3}$. The underlying mechanism of why the smaller void in Form B is not observed in Form C can be proposed from the crystal structures. The mechanism is proposed by comparing the conformational difference of the AZD9898 molecules in Form B and Form C. Each form has two independent molecules in asymmetric unit, namely independent molecule 1 and 2 . An overlay of these independent molecules, shows that the independent molecule 1 of Form $C$ is significantly different from the other respective independent molecules of the other forms. The conformational change consequently allows a hydrogen bond formation of $\mathrm{O}_{103-}$ $\mathrm{H}$...O202 that is not found in Form B. This hydrogen bond replaces the small voids. We also predict that the changes in the unit cell volume upon the heating allows the crystal structure to alter molecular conformation and after the change, it settles into a more efficient packing with a smaller void.

Probing solid-state dehydration and solid-solid phase transformation using in situ $\mathrm{X}$-ray diffraction

As discussed above, the mechanism of the solid-state dehydration from AZD9898 Form A hydrate to Form B, and solid-solid phase transformation from AZD9898 Form B anhydrous to Form $C$ anhydrous can be revealed by using single crystal X-ray structure analysis as single crystallinity was retained upon heating. Therefore, we tried to probe the solidstate dehydration and the solid-solid phase transformation by monitoring the evolution of the lattice cell upon heating using the single crystal $\mathrm{X}$-ray diffractometer. Unit cell parameters were initially measured between 100 and $350 \mathrm{~K}$ with and increment of $10 \mathrm{~K}$. From $350 \mathrm{~K}$, the increment step was reduced to $2 \mathrm{~K}$ to enable observation of more detail around the solidsolid phase transformation.

As can be seen in Fig. 10, the length of $a, b$, and $c$ gradually increases as the temperature rises up to $380 \mathrm{~K}$ (black dashed vertical line). This indicates that the volume of the unit cell expands upon heating. Interestingly, the angles of $\alpha, \beta$, and $\gamma$ remain similar up to $380 \mathrm{~K}$. There is no significant inflection point observed before $380 \mathrm{~K}$. The range of the temperature between 100 and $380 \mathrm{~K}$ represents the temperature range of dehydration from Form A hydrate to Form B anhydrous. The absence of the inflection points below $380 \mathrm{~K}$ support that the solid-state dehydration is an isostructural dehydration process and that no major structural changes are required. Therefore, it is not surprising that AZD9898 Form A hydrate and Form B anhydrous show similarity in their crystal architectures and the ease of dehydration-rehydration between these forms.

A steep inflection point was observed at $380 \mathrm{~K}$ in both lengths and angles of the unit cell lattice parameters. We attribute this as the solid-solid phase transformation of AZD9898 Form B to Form C. This suggests that solid-solid phase transformation is not an isostructural change. Notably, the transition temperature of the solid-solid phase transformation obtained in situ by heating the single crystal in the single crystal $\mathrm{X}$-ray diffractometer is slightly lower than the corresponding temperature obtained from DSC. This difference can be explained by the open and closed systems that were used in the single crystal XRD and DSC, respectively.

A simultaneous XRD-DSC measurement was also carried out in situ to observe the changes of the bulk powder (Fig. 11). The measurement cannot be performed from sub-zero temperatures, which means that Form A hydrate is expected to dehydrate to Form $B$. The absence of the endothermic peak prior the solid- (a)

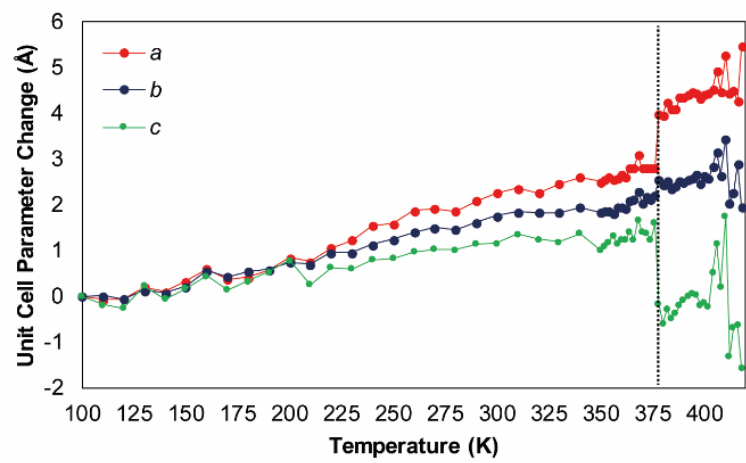

(b)

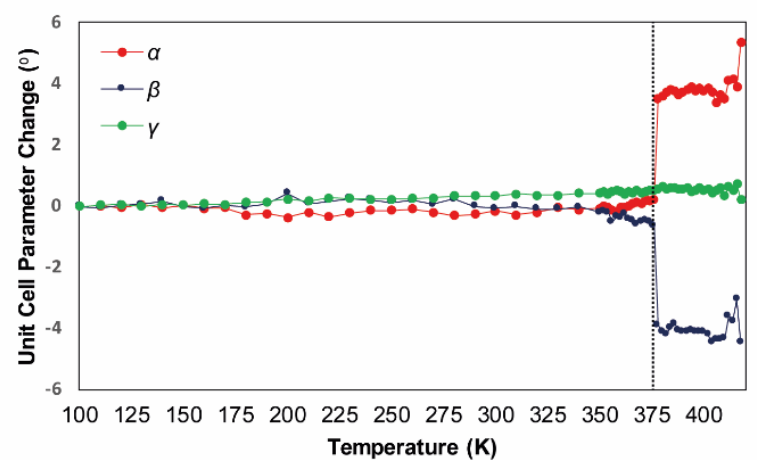

Fig. 10 Change in unit cell parameters in (a) length and (b) angle when increasing the temperature from 100 to $420 \mathrm{~K}$. Black dashed vertical lines indicates the inflection point in unit cell lattice parameters at $380 \mathrm{~K}$ 


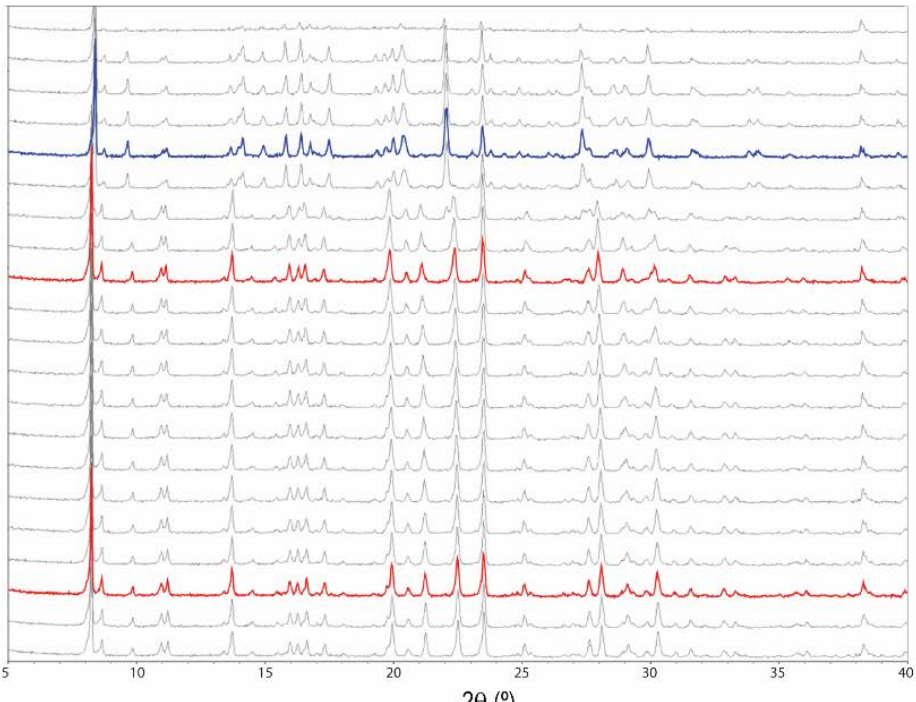

$2 \theta\left({ }^{\circ}\right)$

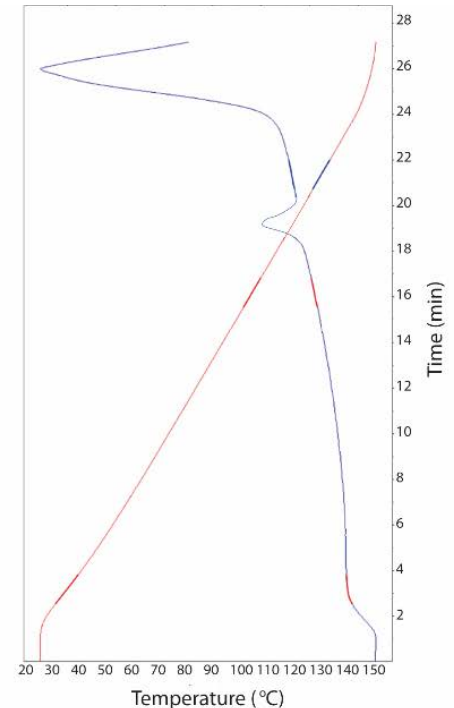

Temperature $\left({ }^{\circ} \mathrm{C}\right)$

Fig. 11 Simultaneous XRD-DSC plot of AZD9898 Form B anhydrous. The right figure shows the DSC plot, and left figure show the change in PXRD pattern with temperature. Red and blue PXRD patterns indicate Form $B$ and $C$, respectively.

solid phase transformation confirms that the sample is already dehydrated and in the Form $B$ anhydrous phase. The PXRD patterns from start of the analysis until slightly below the solidsolid phase transformation temperature are very similar, although cell expansion is observed by the in situ single crystal X-ray measurement.

Above the solid-solid phase transformation temperature, the PXRD pattern no longer possesses similarity with the low temperature PXRD pattern. This again confirms that Form $C$ anhydrous is not isostructural with any of Form $B$ anhydrous or Form A hydrate. It should be emphasized here that the transformation to Form $\mathrm{C}$ does not involve any amorphous or melted phase which is remarkably unusual and allows the structure of Form $C$ to be determined at such high temperature of the same single crystal. The phase transformation of Form B anhydrous to Form $C$ anhydrous upon heating is reversible, meaning that Form $C$ converts back to Form B upon cooling. Thus, Form $B$ and $C$ have an enantiotropic relationship in which one of the forms is stable below the transition temperature, and the other form is stable above the transition temperature.

\section{Conclusions}

In this work, the crystal structures of AZD9898 Form A hydrate and Form $B$ and $C$ anhydrous, were successfully determined by in-situ heating of single crystals of Form $A$ on the X-ray diffractometer. The structural determination of these crystals allows the establishment of the mechanistic aspect of the solidstate dehydration between Form A hydrate and Form B anhydrous, and the solid-solid phase transformation between Form B, and C. The isostructurality between Forms A and B explains the non-stochiometric dehydration-rehydration relationship between these forms which involves minor structural changes to the crystal structure. Solid-solid phase transformation to Form $\mathrm{C}$ requires conformational and hydrogen bond pattern changes leading to a different crystal architecture. The enantiotropic relationship between Form B and $C$ is identified and shows that the transformation is reversible.

\section{Conflicts of interest}

A. P. and O. D. P are AstraZeneca employees and may own stock or stock options.

\section{Notes and references}

¥ Single crystal measurements of AZD9898 Form A hydrate, Form $B$ anhydrous, and Form $C$ anhydrous were performed using the same single crystal with crystal dimension $0.14 \times 0.07 \times 0.01$ $\mathrm{mm}^{3}$.

1 K. R. Morris, U. J. Griesser, C. J. Eckhardt and J. G. Stowell, Adv. Drug Delivery Rev., 2001, 48, 91-114.

2 K. Fujii, H. Uekusa, N. Itoda, E. Yonemochi, K. Terada, Cryst. Growth Des., 2012, 12, 6165-6172.

3 S. Data, D. J. W. Grant. Nat. Rev., 2004, 3, 42-57.

4 O. D. Putra, A. Pettersen, S. O. N. Lill, D. Umeda, E. Yonemochi, Y. P. Nugraha, H. Uekusa, CrytEngComm, 2019, 21, 2053-2057.

5 D. E. Braun, S. R. Lingireddy, M. D. Beidelschies, R. Guo, P. Müller, S. L. Price, S. M. Reutzel-Edens, Cryst. Growth Des., 2017, 17, 5349-5365.

6 A. D. Bond, R. Boese, G. R. Desiraju, Angew. Chem. Int. Ed., 2007, 46, 615-617.

7 K. Suresh, U. B. R. Khandavilli, A. Gunnam, A. Nangia, CrystEngComm, 2017, 19, 918-929.

8 J. Bauer, S. Spanton, R. Henry, J. Quick, W. Dziki, W. Porter, J. Morris, Pharm. Res., 2001, 18,859-866. 
9 O. D. Putra, T. Yoshida, D. Umeda, M. Gunji, H. Uekusa, E. Yonemochi, Cryst. Growth Des., 2016, 16, 6714-6718.

10 R. K. Khankari, D. J. W. Grant, Thermochim. Acta, 1995, 248, 61-79.

11 J. A. Zeitler, K. Kogerman, J. Rantanen, T. Rades, P. F. Taday, M. Pepper, J. Aaltonen, C. J. Strachan, Int. J. Pharm., 2007, 334, 78-84.

12 P. A. Williams, C. E. Hughes, J. Martin, E, Courvoiser, A. B. M Buanz, S. Gaisford, K. D. M. Harris, J. Phys. Chem. C, 2016, 120, 9385-9392.

13 K. Fujii, H. Uekusa, N. Itoda, G. Hasegawa, E. Yonemochi, K. Terada, Z. Pan, K. D. M. Harris. J. Phys. Chem. C, 2010, 114, 580-586.

14 R. Mizoguchi, H. Uekusa, Cryst. Growth Des., 2018, 18, 61426149.

15 H. Mimura, K. Gato, S. Kitamura, T. Kitagawa, S. Kohda, Chem. Pharm. Bull., 2002. 50, 766-770.

16 D. E. Braun, L. H. Koztecki, J. A. McMahon, S. L. Price, S. M Reutzel-Edens. Mol. Pharmaceutics, 2015, 12, 3069-3088.

17 J. Marti-Rujas, A. Morte-Ródenas, F. Guo, N. Thomas, K. Fujii, B. M. Kariuki, K. D. M. Harris, Cryst. Growth Des., 2010, 10, 3176-3181.

18 A. Bajpai, H. S. Schott, T. Pham, K. J. Chen, B. Space, M. Lusi, M. L. Perry, M. J. Zawarotko, IUCrJ, 2016, 3, 430-439.

19 A. Kons, A. Bērzinš, A. Actinš, T. Rekis, S. van Smaalen, A. Mishnev, Cryst. Growth Des., 2019, 19, 4765-4773.

20 K. Fujii, M. Aoki, H. Uekusa, Cryst. Growth Des., 2013, 13, 2060-2066.

21 J. T. J. Freitas, C. C. de Melo, O. M. M. S. Viana, F. F. Ferreira, A. C. Doriguetto, Cryst. Growth Des., 2018, 18, 3558-3568.

22 H. Zhu, J. Xu, P. Varlashkin, S. Long, C. Kidd, J. Pharm. Sci., 2001, 90, 845-859.

23 A. E. Watts, K. Maruyoshi, C. E. Hughes, S. P. Brown, K. D. M. Harris, Cryst. Growth Des., 2016, 16, 1798-1804.

24 O. I. Covaci, D. Samohvalov, C. M. Manta, L. Buhalteanu, A. Barbatu, M. Baibarac, M. Daescu, A. Matea, D. Gherca, J. Mol. Struct., 2019, 1178, 702-710.

25 R. L. Te, U. J. Griesser, K. R. Morris, S. R. Bryn, J. G. Stowell, Cryst. Growth Des., 2003, 3, 997-1004.

26 K. Kobayashi, H. Fukuhara, T. Hata, Y. Ohashi, Chem. Pharm. Bull., 2003, 51, 1356-1362.

27 M. M. A. Rosenschöld, P. Johanneson, A. Nikitidis, C. Tyrchan, H. F. Chang, R. Rönn, D. Chapman, V. Ullah, G. Nikitidis, P. Glader, H. Käck, B. Binn, F. Wågberg, E. Björkstrand, U. Andersson, L. Swedin, M. Rohman, T. Andreasson, E. L. Bergström, F. Jiang, X. H. Zhou, A. J. Lundqvist, A. Malmberg, M. Ek, E. Gordon, A. Pettersen, L. Ripa, A. M. Davis, J. Med. Chem., 2019, 62, 7769-7787.

28 Rigaku Oxford Diffraction, 2015, CrysAlis PRO. Rigaku Oxford Diffraction, Yarnton, England.

29 O. V. Dolomanov, L. J. Bourhis, R. J. Gildea, J. A. K. Howard, H. Puschmann, J. Appl. Cryst., 2009, 42, 339-341.

30 G. M. Sheldrick, Acta Crystallogr., Sect. A: Found. Crystallogr., 2008, 64, 112-122.

31 G. M. Sheldrick, Acta Crystallogr., Sect. C: Struct. Chem., 2015, 71, 3-8.

32 P. Tipduangta, K. Takieddin, L. Fábián, P. Belton, S. Qi, Cryst Growth Des., 2015, 15, 5011-5020.

33 L. Wadsö, N. Markova, Eur. J. Pharm. Biopharm., 2001, 51, 7781.

34 C. F. Macrae, P. R. Edgington, P. McCabe, E. Pidcock, G. P. Shields, R. Taylor, M. Towler, J. van de Streek, J. Appl. Cryst., 2006, 39, 453-457.

35 J. A. Riddick, W. B. Bunger, T. K. Sakano, Techniques of Chemistry: Organic Solvents: Physical Properties, 4th ed. 1986, New York: Wiley-Interscience.
36 D. E. Braun, R. M. Bhardwaj, J. B. Arlin, A. J. Florence, V. Kahlenberg, U. J. Griesser, D. A. Tocher, S. L. Price, Cryst. Growth Des., 2013, 13, 4071-4083.

37 N. Zencirci, E. Gstrein, C. Langes, U. J. Griesser, Thermocim Acta, 2009, 485, 33-42.

38 D. E. Braun, D. A. Tocher, S. L. Price, U. J. Griesser, J. Phys. Chem. B, 2012, 116, 3961-3972.

39 A. L. Spek, J. Appl. Cryst., 2003, 36, 7-13. 

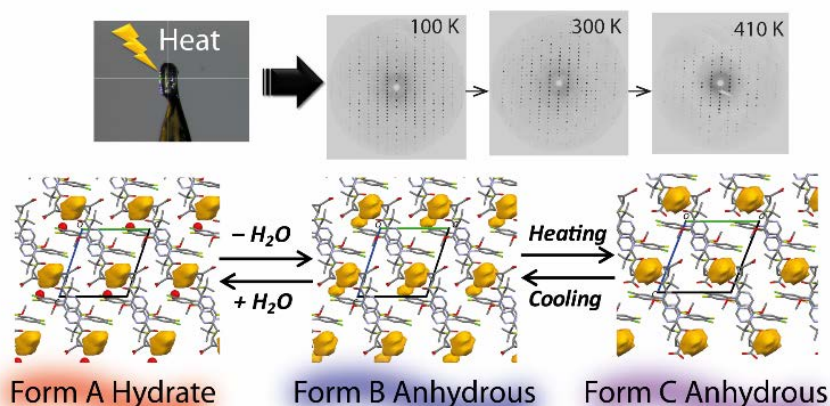

Form A Hydrate Form B Anhydrous Form C Anhydrous 